\title{
Preparation and characterization of hydrophobic platinum-doped carbon aerogel catalyst for hydrogen isotope separation
}

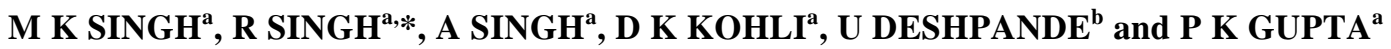 \\ ${ }^{a}$ Nano Functional Materials, Laboratory, Laser Materials Development and Devices Division, \\ Raja Ramanna Centre for Advanced Technology, Indore 452 013, India \\ ${ }^{\mathrm{b}}$ UGC-DAE Consortium For Scientific Research, University Campus, Khandwa Road, Indore 452 013, India
}

MS received 31 May 2013; revised 22 July 2013

\begin{abstract}
We report preparation of hydrophobic platinum-doped carbon aerogel (PtCA) catalyst and its characterization for catalytic exchange reactions between hydrogen isotopes. The PtCA powder was synthesized by sol-gel polymerization method, mixed with colloidal PTFE solution, and coated on Dixon rings to obtain hydrophobic catalyst. The Pt cluster size in PtCA powder was observed to vary from 3 to $5 \mathrm{~nm}$ for a change in resorcinol to alkali molar ratio in synthesis solution from 20 to 200. Transmission electron microscopy of powder showed that the Pt clusters were uniformally dispersed and $\mathrm{Pt}^{\mathbf{0}}$ metallic content estimated by $\mathrm{X}$-ray photoelectron spectroscopy (XPS) was found to be of $\sim 70 \%$. The catalytic activity was found to depend on Pt cluster size and was higher for smaller cluster size. For the smallest achieved Pt cluster size of $3 \mathbf{~ n m}$, catalytic activity of $\sim 0.8 \mathrm{~m}^{3}$ (STP) $\mathrm{s}^{-1} \mathrm{~m}^{-3}$ was obtained for hydrogen isotope exchange in atmospheric pressure conditions.
\end{abstract}

Keywords. Hydrophobic catalyst; carbon materials; sol-gel preparation; nanoparticles; hydrogen isotope exchange.

\section{Introduction}

Liquid phase catalytic exchange (LPCE) reaction between water and hydrogen gas has received considerable attention for both tritium separation and deuterium enrichment. While, tritium separation is required to reduce radiation hazard in heavy water-based nuclear reactors (Kim et al 2007; Popescu et al 2008), deuterium enrichment by LPCE for heavy water production is an attractive process, since it is more energy efficient and environmetal friendly (Izawa 1979) compared with existing 'girder sulphide' and 'ammonia-hydrogen' processes (Aprea 2002). In the LPCE process, hydrogen isotope exchange reactions take place on active sites of platinum $(\mathrm{Pt})$ between gaseous reactants and these sites loose catalytic activity, when covered with liquid water. Therefore, a primary requirement in the catalyst for LPCE process is that it should be hydrophobic (Stevens 1972). Further, to have a good catalytic activity and throughput, a uniform dispersion of Pt particles in a large surface area substrate is required.

Over the years, different support materials such as alumina (Belapurkar et al 1988), silica (Wanke 1985) and activated carbons (Butler 1980) have been investigated for dispersing Pt nanoparticles. Activated carbon supports, in addition to high surface area, help in lowering of activation energy of Pt as compared with alumina and silica

*Author for correspondence (rashmi@rrcat.gov.in) supports (Sagert and Pouteau 1975). However, it has the drawback of inaccessible micropores and mineral impurities. More recently, there has been interest in the use of carbon aerogel as host substate for dispersing $\mathrm{Pt}$, because it offers a good surface area, high level of purity and a possibility of tailoring of the pore sizes (Dawidziuk et al 2009). These Pt-doped carbon aerogels (PtCAs) have primarily been investigated for use as electrocatalyst for fuel cell applications (Kim et al 2008; Guo et al 2011). It is also pertinent to note that several synthesis methods like conventional wet impregnation, electroless deposition and sputtering have been used for uniformally dispersing Pt nanoparticles on high surface area carbon supports. While the wet impregnation and electroless deposition do not provide adequate control of particle size and distribution, sputtering method poses technical challenges for mass scale production (Esmaeilifar et al 2010).

We describe in this paper, the use of in situ sol-gel method for dispersing platinum on carbon aerogel. This method not only leads to a uniform dispersion of platinum in carbon aerogel, but also provides some control on the particle size by adjusting the process parameters like $\mathrm{pH}$, pyrolysis temperature and concentration. This control on particle size is desirable because a lower mean particle size for Pt, helps in achieving the same catalytic activity with less quantity of Pt thus, reducing the cost of the catalyst. PtCA powder was synthesized by sol-gel polymerization method, mixed with colloidal PTFE solution, and coated on Dixon rings to obtain hydrophobic 
catalyst. After describing the synthesis and characterization of the catalyst, we also present results of its use for $\mathrm{H} / \mathrm{D}$ exchange reactions in atmospheric pressure conditions.

\section{Experimental}

\subsection{Catalyst preparation}

PtCA powder was prepared by ambient pressure drying process reported earlier by us (Singh et al 2010). In this process, platinum precursor was added to the synthesis solution before polymerization of gel. As described in our previous work, PtCA powder was synthesized by polycondensation of resorcinol (R) and furfuraldehyde in isopropyl-alcohol. Hydrogen-hexachloroplatinate was used as platinum precursor. Potassium hydroxide $(\mathrm{KOH})$ was added to the solution in different molar ratios to obtain $\mathrm{R} / \mathrm{KOH}$ of $20,50,100,150$ and 200 . The solution was kept in a sealed vial at $60{ }^{\circ} \mathrm{C}$ for $72 \mathrm{~h}$ for complete gelation. The gel was dried under ambient conditions and pyrolysed in argon atmosphere at $500{ }^{\circ} \mathrm{C}$ employing heating rate of $4{ }^{\circ} \mathrm{C} / \mathrm{min}$ to obtain $7 \%$ PtCA powder.

Catalyst was prepared by coating the slurry of PtCA powder and PTFE on Dixon ring support to achieve hydrophobicity and mechnical strength. PTFE binder was selected among other hydrophobic binders like SDBC and PVDF, since it has better hydrophobic property owing to high contact angle $\left(108^{\circ}\right)$, high temperature sustainability and long time stability (Huang and Meng 2010). Coated Dixon rings were cured at $370^{\circ} \mathrm{C}$ in argon atmosphere to obtain hydrophobic catalyst (contact angle $\sim 150^{\circ}$ ).

\subsection{Physiochemical characterizations}

The surface morphology of PtCA powder and the coated film of PTFE and PtCA on Dixon rings were examined using scanning electron microscope (SEM) (Carl Zeiss, Zigma). Surface area and pore volume of PtCA powder were measured by BET surface area measurement system (Micromeritics ASAP2020). Pt cluster size and its distribution on carbon aerogel support were analysed by transmission electron microscopy (TEM Philips CM 200) and X-ray (RIGAKU) diffractometer. The distribution of Pt cluster size was estimated from histograms obtained using TEM images. X-ray photoelectron spectroscopy (XPS) analysis was carried out on ESCA lab250 spectrometer to determine the chemical states of Pt.

\subsection{Measurement of catalytic activity}

Catalytic exchange reaction between deuterated water and hydrogen occurs according to the following reversible reaction

$$
\mathrm{H}_{2}+\mathrm{HDO} \stackrel{\text { Catalyst }}{\longleftrightarrow} \mathrm{HD}+\mathrm{H}_{2} \mathrm{O} .
$$

The complete reaction mechanism is well documented in literature (Sagert and Pouteau 1974). The catalytic activity was measured in terms of volume transfer rate, Kya, as described in literature (Butler 1980), which defines the net amount of deuterium transferred from isotopicallyenriched liquid water to the hydrogen stream in a unit volume of packed catalyst bed under conditions of unit displacement. Kya is expressed as $\mathrm{m}^{3}$ (STP) $\mathrm{s}^{-1} \mathrm{~m}^{-3}$ of catalyst bed, and can be evaluated using follwing expression

$$
\text { Kya }=\left(F_{\mathrm{H}_{2}} * A / V\right)(-\ln (1-\eta)),
$$

where $F_{\mathrm{H}_{2}}$ is the superficial flow rate of hydrogen gas, $A$ and $V$ the cross-sectional area and volume of catalyst packed bed, respectively, and $\eta$ the isotope exchange efficiency.

Catalyst performance tests were performed in a glass column with internal diameter of $25 \mathrm{~mm}$ and height of $1000 \mathrm{~mm}$. The column was filled with coated Dixon rings to a height of $220 \mathrm{~mm}$. Water having $2000 \mathrm{ppm} \mathrm{D/H}$ was fed at the top of the column and natural hydrogen gas ( $50 \mathrm{ppm} \mathrm{D/H}$ ) was fed from bottom of the column. The operating conditions were as follows: temperature $-32{ }^{\circ} \mathrm{C}$, $\mathrm{H}_{2} \mathrm{O}$ flow rate $-3 \mathrm{~kg} / \mathrm{h}$ and $\mathrm{H}_{2}$ gas flow rate $-14 \mathrm{lpm}$. The $\mathrm{D} / \mathrm{H}$ concentration in the hydrogen gas at inlet and outlet of the column were analysed with $\mathrm{D} / \mathrm{H}$ mass spectrometer. Experimental conditions were selected in such a way that it leads to a concentration of $\mathrm{D} / \mathrm{H}$ which will be in the measurable range with the mass spectrometer.

\section{Results and discussion}

The morphology of PtCA powder and the coated Dixon ring catalyst are shown in SEM images (figure 1(a) and (b)). Figure 1(a) shows highly porous open structure of PtCA powder. SEM image of coated Dixon ring film shows that PTFE covers the open structures of PtCA powder (figure $1 \mathrm{~b}$ ). The PTFE film is permeable to gaseous reactants, but prevents water penetration to active $\mathrm{Pt}$ sites. Surface area and pore volume of PtCA powder were measured to be $605 \mathrm{~m}^{2} / \mathrm{g}$ and $0.24 \mathrm{~cm}^{3} / \mathrm{g}$, respectively.

In figure 2, we show the representative TEM images and corresponding histograms of particle size distribution for PtCA powder prepared with varying molar ratios of $\mathrm{R} / \mathrm{KOH}$. Figure 2(a) shows highly dispersed Pt nanoparticles with almost no agglomeration for $\mathrm{R} / \mathrm{KOH}$ of 100 . It has mean particle size of $3.01 \mathrm{~nm}$ with standard deviation of $0.39 \mathrm{~nm}$. Bigger particle size and agglomeration were observed for lower and higher values of R/KOH (figure 2(b) and (c)).

Figure 3 shows XRD spectra of PtCA powder, where the major diffraction peaks of Pt can be seen at $2 \theta=39.6^{\circ}$, $46 \cdot 1^{\circ}, 67 \cdot 4^{\circ}, 81 \cdot 2^{\circ}$ and $85.6^{\circ}$ corresponding to the reflection planes of (1 11 1), (2 00 ), (2 20 ), (3 11 1) and (2 2 2). The peaks are in consistent with the face-centered cubic ( $f c c)$ structure of platinum (JCPDS card no. 04-0802). The $f c c$ phase of crystalline platinum demonstrates a successful 

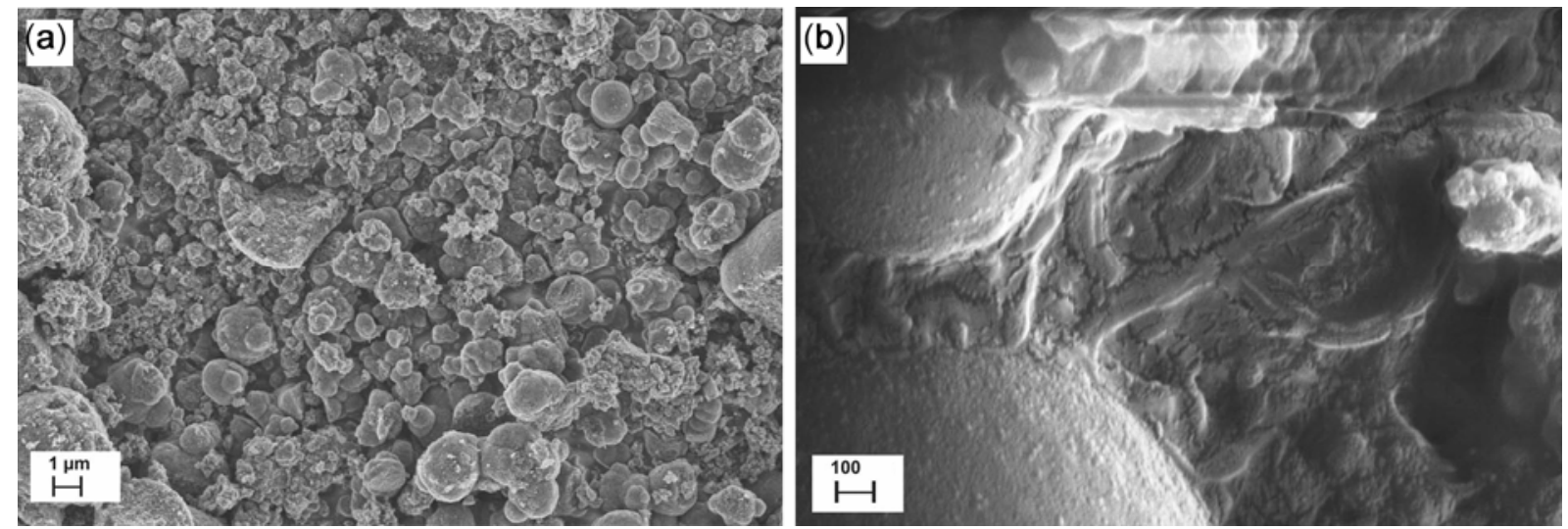

Figure 1. SEM image of (a) PtCA powder and (b) coated film of PTFE and PtCA on Dixon ring.
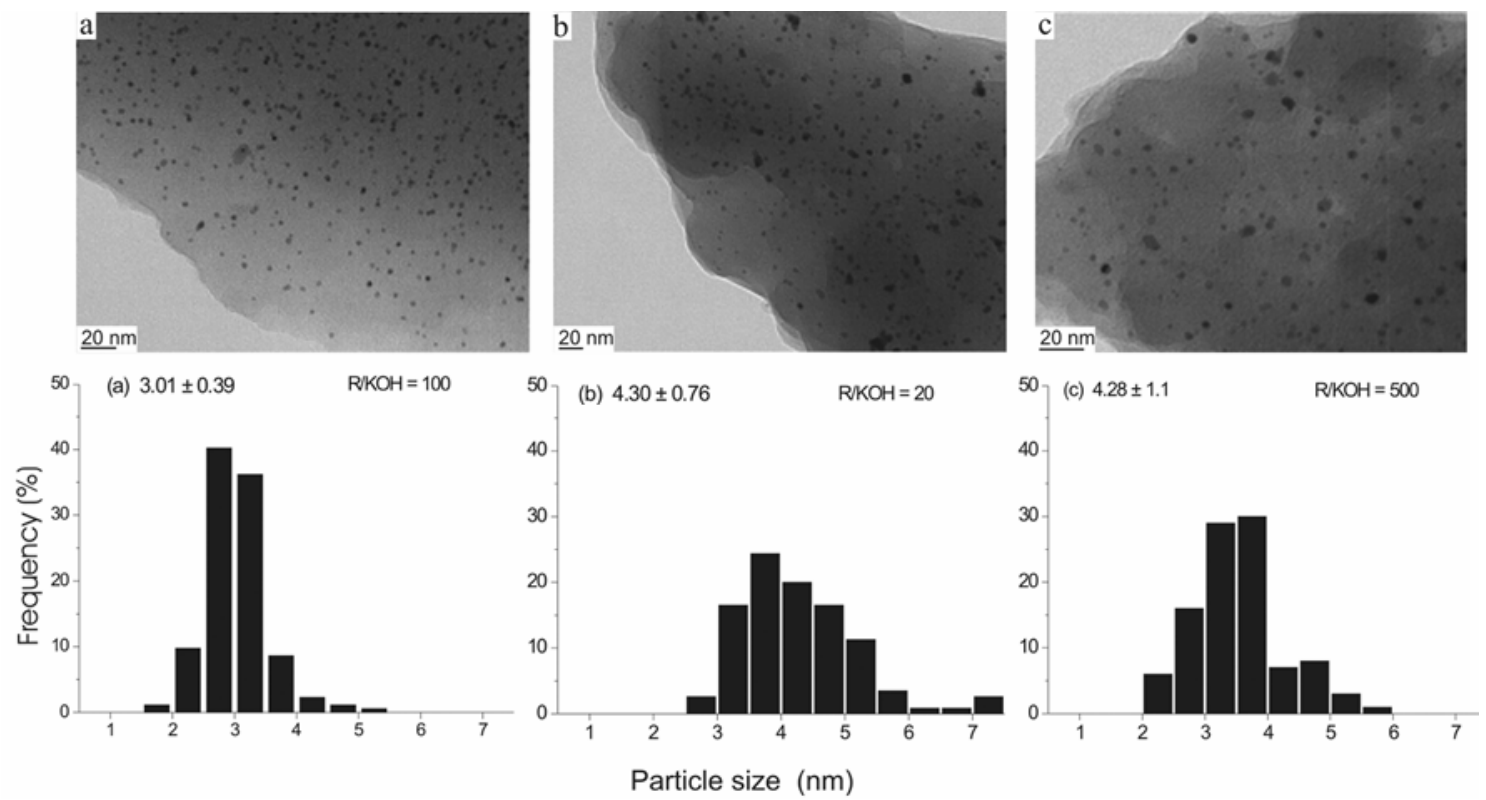

Figure 2. (a)-(c) TEM images of Pt cluster on carbon aerogel and their corresponding histograms for varying molar ratios of $\mathrm{R} / \mathrm{KOH}$.

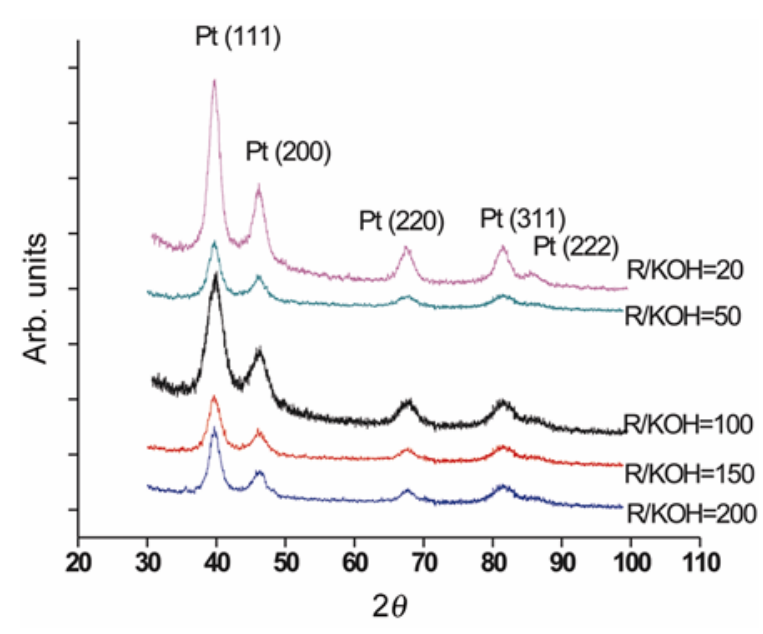

Figure 3. XRD spectra of PtCA powder for varying molar ratios of $\mathrm{R} / \mathrm{KOH}$.

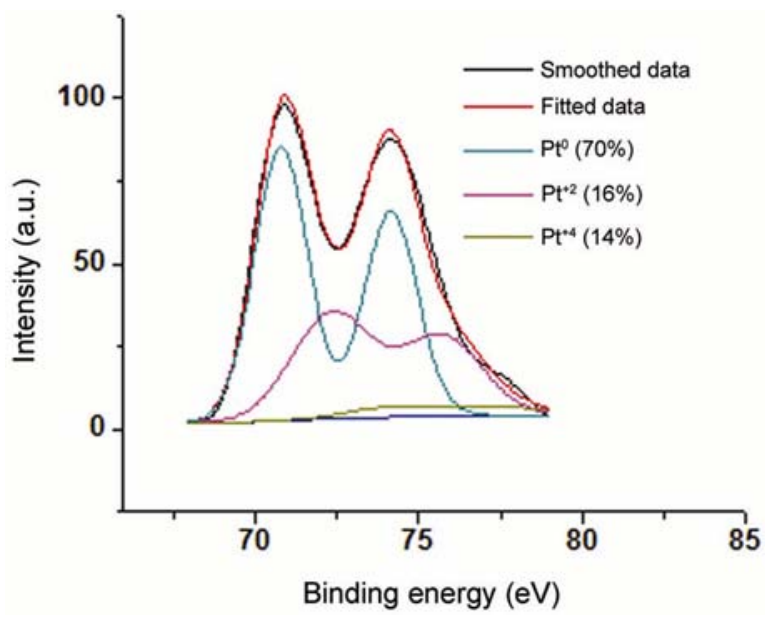

Figure 4. XPS spectra for Pt $4 f$ region with relative intensities for different Pt states. 
Table 1. Catalytic activity in terms of Kya for different Pt cluster sizes having varying $\mathrm{R} / \mathrm{KOH}$ molar ratios.

\begin{tabular}{ccc}
\hline R/KOH ratio & Pt cluster size $(\mathrm{nm})$ & Kya $\left(\mathrm{m}^{3}(\mathrm{STP}) \mathrm{s}^{-1} \mathrm{~m}^{-3}\right)$ \\
\hline 20 & $4 \cdot 3 \pm 0.76$ & 0.51 \\
50 & $3.7 \pm 0.68$ & 0.67 \\
100 & $3.01 \pm 0.39$ & 0.81 \\
150 & $3.9 \pm 0.63$ & 0.61 \\
200 & $4.5 \pm 1 \cdot 1$ & 0.47 \\
\hline
\end{tabular}

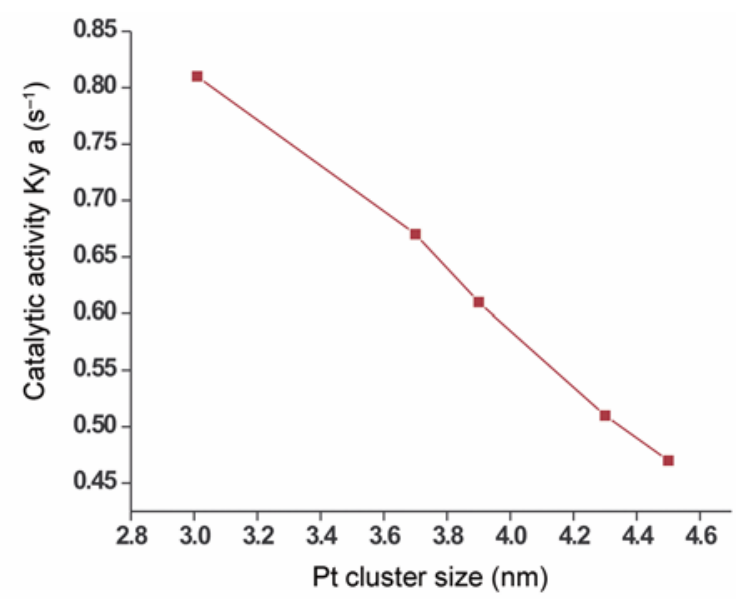

Figure 5. A plot of Pt particle size vs catalytic activity.

reduction of platinum precursor to the metallic form. Particle size calculated using the Scherrer equation is in close agreement with the results obtained from TEM analysis.

In figure 4, we show XPS spectra for Pt $4 f$ region, which was de-convoluted to three pairs of doublets by XPSPEAK software with their relative intensities. Reasonably high metallic $\mathrm{Pt}^{0}$ content $\sim 70 \%$, suggests that synthesis process effectively reduces platinum salt into $\mathrm{Pt}^{0}$ metallic state, which is desirable for good catalytic activity (Hu et al 2011).

\subsection{Catalytic performance of the hydrophobic catalysts}

Measurement of catalytic activity was carried out under test conditions as explained earlier in $\S 2.3$. Results of the measurements made on catalytic activity of catalysts prepared using different molar ratios of $\mathrm{R} / \mathrm{KOH}$ are presented in table 1.

The results show that the best activity is obtained for $\mathrm{R} / \mathrm{KOH}$ ratio of 100 , for which the Pt cluster size is lowest (3.01 $\pm 0.39 \mathrm{~nm})$. The result of higher activity for smaller particle size (figure 5) is in agreement with previous reports (Hu et al 2011), where a similar dependence of activity on Pt cluster size has been reported.

Measurements of catalytic activity over a period of one month at atmospheric pressure conditions show that loss in activity was less than $5 \%$.

\section{Conclusions}

Hydrophobic catalyst for hydrogen isotope exchange reactions was prepared using PtCA powder and PTFE binder. This study shows that resorcinol to alkali ratio in synthesis solution has effect on Pt cluster size and lowest Pt cluster size of $3.01 \pm 0.39 \mathrm{~nm}$ was obtained for $\mathrm{R} / \mathrm{KOH}$ of 100. Physiochemical characterization shows that PtCA powder has high surface area, well-dispersed Pt nanoparticles and high $\mathrm{Pt}^{0}$ metallic state. This catalyst has shown good catalytic activity and stable performance in atmospheric conditions for LPCE process. The catalytic activity was found to depend on Pt cluster size and was higher for smaller cluster size. For the smallest achieved Pt cluster size of $3 \mathrm{~nm}$, catalytic activity of $\sim 0.8 \mathrm{~m}^{3}$ (STP) $\mathrm{s}^{-1} \mathrm{~m}^{-3}$ was obtained for hydrogen isotope exchange in atmospheric pressure conditions.

\section{Acknowledgements}

We would like to thank Shri A Bhomick and their colleagues at HWP, Baroda, for carrying out the catalytic activity measurements and Shri A K Agarwal and Shri Rajnish Prakash HWB for their encouragement and support. We also thank Shri Gopal Mohod, RRCAT, Indore, for his assistance.

\section{References}

Aprea J L 2002 Hydrogen Energy 27741

Belapurkar D, Gupta N M and Iyer R M 1988 Appl. Catal. 431

Butler J P 1980 US Patent 4228034

Dawidziuk M B, Carrasco-Marin F and Morena-Castilla C 2009 Carbon 472679

Esmaeilifar A, Rowshanzamir S, Eikani M H and Ghazanfari E 2010 Energy 353941

Guo Z, Zhu H, Zhang X, Wang F, Guo Y and Wei Y 2011 Bull. Mater. Sci. 34577

Hu S, Hou J, Xiong L, Weng K, Yang T and Luo Y 2011 Sep. Purif. Technol. 77214

Huang F and Meng C 2010 Int. J. Hydrogen Energy 356108

Izawa H 1979 J. Nucl. Sci. Technol. 16741

Kim H J, Kim W, Jin T, Park H S and Suh D J 2008 Carbon 46 1393

Kim K R, Lee M S, Paek S, Yim S P, Ahn D H and Chung H 2007 Sep. Purif. Technol. 54410

Popescu I, Ionita Gh, Stefanescu I, Varlam C, Dobrinescu D and Faurescu I 2008 Fusion Eng. Des. 831392

Sagert N H and Pouteau R M L 1974 Can. J. Chem. 522960

Sagert N H and Pouteau R M L 1975 Plat. Met. Rev. 1916

Singh R, Khardekar R K, Kohli D K, Singh M K, Srivastava H and Gupta P K 2010 Mater. Lett. 64843

Stevens W H 1972 Canadian Patent 907292

Wanke S E 1985 US Patent 4536488 